\title{
A review of the book Problem-Solving and Selected Topics in Number Theory:In the Spirit of the Mathematical Olympiads, Springer, 2011, xiii+324 pp., ISBN 978-1-4419-0494-2
}

\author{
by Sotirios E. Louridas and Michael Th. Rassias
}

The book under review is an excellent and very friendly introduction to number theory through theory, methods and beautiful problems. It contains all the necessary material corresponding to an undergraduate level course in number theory and it also includes an invaluable selection of problems for the preparation of students for mathematical Olympiads and international contests in mathematics.

The book starts with fundamental concepts and gradually presents more advanced topics, which are presented in a very approachable way. A Foreword by the well-known number theorist Preda Mihailescu (University of Goettingen, Germany) is featured at the beginning of the book. In this Foreword a conjecture of Michael Th. Rassias is presented along with its connections to a number of famous problems in Number Theory.

There are twelve chapters organized in sections, dealing with Arithmetic Functions, Perfect Numbers, Fermat Numbers, Congruences, Quadratic Residues, the Riemann-zeta Function, Dirichlet Series and several other topics. The definitions and relevant theorems are rigorously presented and the reader is introduced in the field of number theory in such a manner that allows him/her to go through the material with great pleasure. Additionally, all the theorems stated in this book are followed by very detailed proofs. 
The Appendix provided in the final part of the book, consists of an important set of classical and deep results which will be an inspiring motivation for students to do research. In this section, the Prime Number Theorem is proved in detail and in a self-contained manner.

The book contains a set of 105 problems, along with their comprehensive solutions. The problems are very intriguing, as almost each one of them is based on a different new idea. Even simple arguments, such as parity properties, divisibility criteria, or the factorization principle, are applied in an ingenious manner in order to obtain interesting results. Some of the problems are selected from Putnam Competitions, IMOs, IMO Shortlists, or from wellknown mathematical journals including the American Mathematical Monthly and the Crux Mathematicorum.

Another key feature which played an integral role for making this book so successful is the fact that the author was a top problem-solver at international mathematical competitions, having obtained several awards and medals.

The book is of high quality and belongs to every library.

Cristinel Mortici

"Valahia" University, Târgovişte, Romania, and

Academy of Romanian Scientists, Romania 